ApplicationForum

\title{
Monitoring of insulin granule packaging in live cells using homoFRET-FP detection
}

\author{
N.Y. Y'i, Q. He', T.B. Caligan', G.R. Smith', L.J. Forsberg'2, J.E. Brenman² and J. Z. Sexton' \\ ${ }^{1}$ North Carolina Central University, Durham, NC ${ }^{2}$ UNC Chapel Hill School of Medicine, Chapel Hill, NC
}

\section{Introduction}

This application note presents a novel high-throughput screening compatible cell-based assay to monitor insulin granule packaging. It uses changes in fluorescence polarization (FP) due to homoFRET that is detected by the PHERAstar ${ }^{\circledR}$ microplate reader.

Diabetes mellitus is characterized by disruption of metabolism that stems from resistance to insulin or poor insulin secretion. In 2014 nearly $10 \%$ of the U.S. population suffered from diabetes of which $90 \%$ were type-2 diabetes. Because of the high prevalence and the equally high financial burden associated with treatment it remains a focus to find new therapeutics.

\section{Assay Principle}

The application exploits that a fluorophore at a high local concentration leads to FRET between the same type of fluorophores (homoFRET). Furthermore, if polarized light is applied as the excitation light it will become randomized as homoFRET occurs between adjacent fluorophores. It was reasoned that a homoFRET-FP approach would monitor the extent of packing of mature insulin into dense core granules (Figure 1). The assay is based on the preproinsulin-mCherry (PPI-mCherry) system that uses mCherry as a fluorophore coupled to the precursor preproinsulin.

\section{Materials \& Methods}

Insulin producing rat insulinoma (INS-1) cells were transfected with the Preproinsulin (PPI) mCherry Reporter Construct. The V-ATPase inhibitor bafilomycin blocks vacuole maturation and was used to prevent insulin granule formation. The cells were allowed to settle for $48 \mathrm{~h}$ and were subsequently exposed to bafilomycin for $4 \mathrm{~h}$. Measurement of mCherry homoFRET-fluorescence polarization was performed on BMG LABTECH's PHERAstar.

\section{Results \& Discussion}

To validate the homoFRET-FP approach, cells were treated with bafilomycin to block insulin granule formation (Figure 2). Increasing concentrations of bafilomycin reduce granularity and increase $\mathrm{MP}$ values due to reduced homo-FRET-randomization of the emission light. This is explained by low packaging of insulin. At low bafilomycin concentrations and accordingly high granularity, homo-FRET between tightly packed mCherry-coupled insulin leads to randomization of the emission light and low FP values.

\section{Conclusion}

These results establish a novel cell-based FP biosensor to identify compounds that modify insulin granule packaging. This assay technology combined with the PHERAstar reader may serve as a new method for assessing protein-protein interactions in live cell systems.

\section{References}

${ }^{1}$ Yi NY et al. (2015) Development of a Cell-Based Fluorescence Polarization Biosensor Using Preproinsulin to Identify Compounds That Alter Insulin Granule Dynamics. Assay Drug. Dev. Technol. 13(9): 558-569.

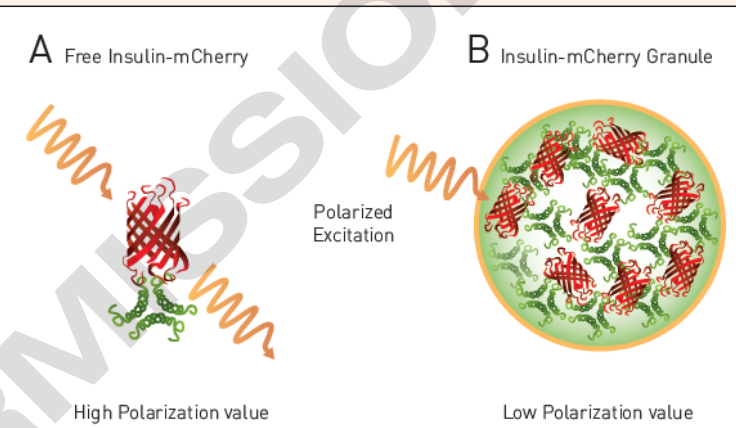

Fig. 1: homoFRET-FP to detect packaging of insulin in dense core granules in live cells. A) Free insulin-mCherry with polarized excitation will exhibit conserved polarization and relatively high MP signal. B) Within dense core granule polarized light will exhibit homoFRET, randomized polarization and a decrease in MP signal ${ }^{1}$.

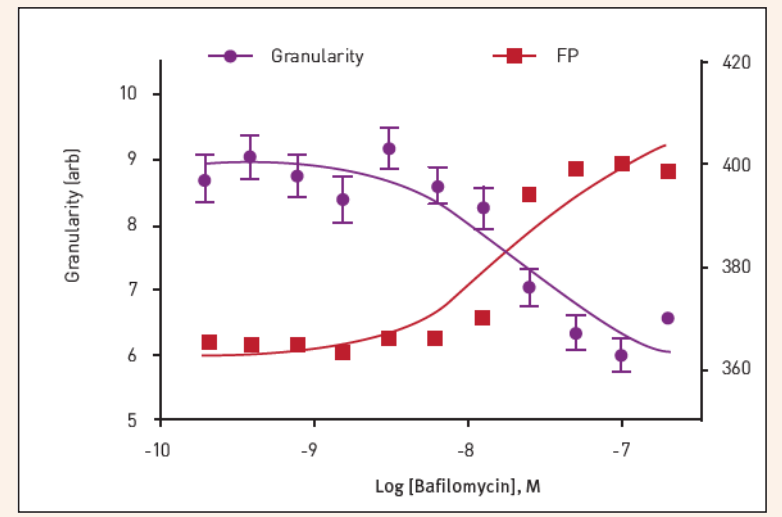

Fig.2: Dose response to Bafilomycin in Insulin Granule Packing Assay. Analysis shows an anti-correlation between homoFRET-FP signal and mCherry granularity ${ }^{1}$. 